REDES- Revista hispana para el análisis de redes sociales

Vol.20,\#3, Junio 2011

http://revista-redes.rediris.es

\title{
El capital social y las redes personales en el estudio de las trayectorias laborales*
}

\author{
Pedro López-Roldán - QUIT, Departamento de Sociología ${ }^{1}$ (UAB) \\ Vanessa Alcaide Lozano - Departamento de Sociología y Análisis de las \\ Organizaciones $^{2}(\mathrm{UB})$
}

\section{Resumen}

En este artículo se pretende aplicar el enfoque del Capital Social al estudio de las Trayectorias Laborales de la población que reside en Cataluña. Esta aplicación se realiza a través de dos planteamientos o perspectivas. La primera se centra en las características atributivas que configuran el entorno laboral de la población estudiada. A través de esta perspectiva se identifican los tipos de Trayectorias Laborales que se dan tanto en la población autóctona como en la población inmigrante. La segunda perspectiva se centra en las características relativas al entorno relacional de esta población desde una visión puramente reticular. Esta segunda visión se lleva a cabo a partir del estudio de las redes personales de la población autóctona e inmigrante. La articulación de estas dos perspectivas a partir del tratamiento de dos bases de datos de diferente naturaleza y la aplicación de los elementos que constituyen el enfoque del Capital Social nos permite aproximarnos al papel que juegan las relaciones entendidas como recurso en el marco de la experiencia laboral y en el contexto del mercado de trabajo catalán. Para finalizar se identifican los mecanismos relacionales que actúan en este proceso de trayectoria en términos de Cohesión e Integración Social. Estos mecanismos se hacen operativos a partir del estudio de las relaciones de tipo Bonding Social Capital y Bridging Social Capital.

Palabras clave: Capital social - Trayectorias laborales - Construcción de tipologías - Perspectiva atributiva - Perspectiva reticular - Cohesión social - Integración social - Análisis de redes personales - Muestra continua de vidas laborales.

\begin{abstract}
This paper attempts to apply the Social Capital approach to the study of the Labour Market Careers of the population residing in Catalonia. This application is done through two approaches or perspectives. The first one focuses on the attributive characteristics that shape the working environment of the population. Through this perspective we identify types of Labour Market Careers that are given both in the autochthonous and the immigrant population. The second one tries to analyze the relational environment of this population from a purely reticular vision. This second view is held by studying the configuration of personal networks. The articulation of these two views and the application of the elements that constitute the Social
\end{abstract}

\footnotetext{
* Este artículo se inscribe en un proyecto de investigación, subvencionado por el Ministerio de Ciencia e Innovación español dentro del VI Plan Nacional de Investigación Científica, Desarrollo e Innovación Tecnológica 2008-2011 (referencia CSO2008-01470) y llevado a cabo en el Centro de Estudios Sociológicos sobre la Vida Cotidiana y el Trabajo (QUIT, http://quit.uab.cat/).

1 Enviar correspondencia a: <Pedro.Lopez.Roldan@uab.cat>.

2 Enviar correspondencia a: < valcaide@ub.edu>.
} 
Capital approach allow us to come closer to the role of relations considered as resources in the context of working experience and in the context of Catalan labour market. Finally, we identify the relational mechanisms acting in this career process in terms of Social Cohesion and Integration. These mechanisms become operative through the study of Bonding Social Capital and Bridging Social Capital types of relations.

Key words: Social Capital - Labour Market Careers - Construction of Typologies Attributive Perspective - Network Perspective - Social Cohesion - Social Integration - Personal Networks Analysis - Continuous Sample of Working Lives.

\section{Introducción}

Son diversos los autores que desde una perspectiva tanto atributiva (Requena, 1991; Toledo y Bastourre, 2006) como reticular (Granovetter 1973, 1974; Burt 2003) han puesto de manifiesto la importancia de las relaciones sociales para potenciar el acceso y la movilidad en el mercado de trabajo. Estas relaciones pueden proporcionar oportunidades laborales que derivan tanto del entorno familiar más próximo como de contextos menos informales en los que se interacciona con individuos más o menos semejantes en términos atributivos. En función de estas semejanzas o disimilitudes atributivas, los individuos pueden manejar informaciones que resultan fundamentales en el contexto de interacción. Este intercambio y difusión de la información que circula por la red puede generar o potenciar oportunidades interesantes para mejorar la posición en el mercado de trabajo.

Por un lado, nos interesa conocer cómo se generan las sinergias en la interacción en el contexto de los diferentes tipos de Trayectorias Laborales ( $T L$ ) que se identifican en la población residente en Cataluña. Por otro, analizar cómo en el marco de estos tipos de trayectorias funcionan los mecanismos relacionales de Cohesión Social (CoS) e Integración Social (InS). Para ello, en primer lugar se expone qué se entiende por Capital Social (CS) desde una perspectiva tanto atributiva como reticular y cómo se articulan los mecanismos relacionales de CoS e InS en el marco de las TL.

En segundo lugar se presentan los colectivos que serán estudiados y las dos bases de datos utilizadas. El objetivo es articular datos de carácter puramente atributivo con datos que derivan de la aplicación de un cuestionario de redes personales, y por tanto de carácter reticular. 
En tercer lugar se presenta la dimensionalización de los conceptos utilizados a través de una propuesta de medición. Para finalizar se analizan los resultados que derivan de la aplicación de diversas técnicas de análisis multivariado a través de las cuales se construyen las TL. En este contexto se analizan, para cada uno de los colectivos estudiados, sus niveles de CoS e InS. Estos mecanismos relacionales ayudan a comprender la posición laboral de los individuos estudiados y dan pistas sobre los recursos de los que disponen en sus redes personales para mantener 0 mejorar su posición en el mercado de trabajo.

\section{El enfoque del Capital Social en el contexto de la Trayectoria Laboral}

\subsection{La multidimensionalidad del concepto de Capital Social y los tipos de relaciones}

Una de las primeras definiciones del concepto de CS data de 1916 y fue acuñada por L. J. Hanifan. En su escrito, el autor ponía de manifiesto la importancia del compromiso comunitario para el apoyo a la democracia y el desarrollo (Hanifan, 1916). Durante gran parte del siglo XX el concepto ha sido reinventado varias veces, puesto que ninguna de las definiciones propuestas solidificó entre los estudiosos del momento. No es hasta finales de los años 80 que se produce la consolidación del concepto. Este proceso ha venido de la mano de tres autores que han proporcionado las tres visiones clásicas o pilares del concepto de CS desde diferentes disciplinas, entre ellas, la sociología. Estos autores son James S. Coleman, Pierre Bourdieu y Robert D. Putnam.

La definición estructural o de carácter relacional de CS viene dada por los trabajos de Bourdieu y Coleman. En sus definiciones, ambos hacen referencia al CS como un elemento que deriva de la pertenencia o la participación en redes sociales (Herreros, 2002). Bourdieu se refiere al CS como un agregado de recursos relacionales, actuales o potenciales, que se desprenden de la pertenencia a una red de relaciones duradera (Bourdieu, 1986). Coleman pone el énfasis en la función que el CS desempeña en el marco de la estructura y de la acción de los individuos (Coleman, 1988). Teniendo en cuenta el enfoque estructuralista, aunque más orientada hacia una visión culturalista, se encuentra la definición de CS de Robert D. Putnam (2003). Este autor entiende el CS como aquellos aspectos de la organización social, como son las redes, las normas de confianza y reciprocidad, que conforman una cualidad de los grupos o del conjunto de la sociedad y que permiten la cooperación (Putnam, 1993). 
Tal y como se expresa en la literatura sobre CS (Lozares, 2003; Herreros, 2002; Burt, 1997; Woolcock y Narayan, 2000; Lin, 2001; Field, 2008) el concepto puede ser abordado desde diversos enfoques de tal manera que en cada uno de ellos se pone el énfasis en distintos ejes que cortan en varias dimensiones la idea básica que envuelve al concepto, como recurso individual y/o colectivo (Portes, 1998), como perspectiva estructuralista o culturalista (Herreros, 2002), según los tipos de relaciones o de CS (Woolcock y Narayan, 2000), etc.

Partiendo de la idea de recurso, de carácter actual y potencial, que se genera en el marco de la interacción y por tanto en el contexto estructural de una red social, cabe señalar dos elementos que se consideran fundamentales para la comprensión de la idea de CS y para facilitar la tarea de realización del trabajo empírico. Estos dos elementos van en la línea de lo que Frane y Roncevic (2003) recogen al hablar de la identidad del CS segmentada en un genotipo y sus fenotipos.

Consideramos que aquello que se entiende por CS puede expresarse a partir de esta identidad segmentada del concepto. Se parte de la idea de que el CS se constituye por dos elementos: uno interno y otro externo. En el elemento interno, o núcleo duro, encontramos la referencia a la interacción y concretamente a la forma e intensidad que esta puede adquirir. En el elemento externo, se añade el matiz sobre la composición de aquellos factores que circulan por la estructura de la red. Este segundo elemento está más vinculado al contenido de la interacción y al marco de la misma, como es el mercado de trabajo en este caso.

En el elemento interno del CS podemos diferenciar dos tipos de interacciones que proporcionan determinadas oportunidades o recursos en función de la posición que el individuo tiene en la estructura de la red. Estos dos tipos conforman la tipología de CS y son utilizados aquí desde una perspectiva relacional. El primero se refiere a aquellas formas de interacción "de unión" que dan lugar al Bonding Social Capital. El segundo hace referencia a las relaciones "que tienden puentes" y generan Bridging Social Capital (Woolcock y Narayan, 2000; Field, 2008).

La idea de Bonding Social Capital nos permite hacer operativo el primero de los mecanismos estudiados, el de la CoS (Lozares et al., 2011). La CoS es la situación en la cual la estructura de interacción entre los individuos de un colectivo previamente definido se da de forma dirigida hacia el interior del grupo. Se trata pues de relaciones horizontales de carácter fuerte que van sujetas a un elemento atributivo en términos de homofilia (Lin, 2001), es decir, entre individuos que son atributivamente similares (ya sea por su nacionalidad, por su posición en el mercado de trabajo, por sus niveles de cualificación, etc.). 
A través del Bridging Social Capital se hace operativo el segundo de los mecanismos estudiados, el de la InS (Lozares et al., 2011). En situación de InS la interacción que se da entre los individuos inmersos en la estructura de una red está dirigida hacia el exterior del colectivo previamente definido, es de carácter débil y puede ser tanto horizontal como vertical. En términos atributivos recogen la idea de heterofilia (Lin, 2001) en la cual las relaciones se dan entre individuos atributivamente diferentes (Figura 1). Esto implica que los individuos pertenecen a entornos $\mathrm{y} / \mathrm{o}$ colectivos diferentes y que las relaciones pueden ser menos frecuentes o intensas. Aún así los contenidos que circulan por la estructura de la red pueden ser más variados, aumentando la probabilidad de que la información disponible no sea redundante para los individuos (Granovetter, 1973, 1974).

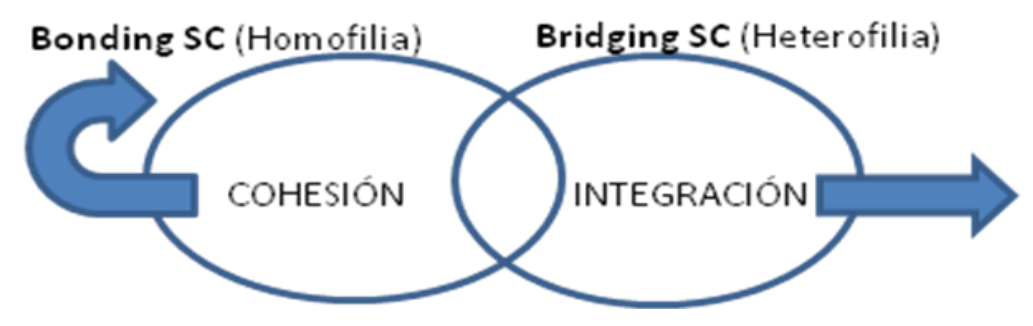

Figura 1. Tipos de Capital Social y sus mecanismos relacionales en conjuntos definidos.

\subsection{La Trayectoria Laboral como marco para la activación de recursos}

La visión que se tiene en este artículo sobre el mercado de trabajo es la de un complejo entramado social que no sólo implica la interacción entre individuos y oportunidades que se generan en la estructura (Canteros y Espinoza, 2001), sino que además plantea interacciones a diferentes niveles micro, meso y macro (Lozares, 1996; Molina, 2001, 2004). Estas interacciones se dan en segmentos diferenciados del mercado de trabajo y en un contexto económico globalizado (López-Roldán, 1996b; Alós-Moner, 2008).

El modelo actual de mercado de trabajo español viene caracterizado por un aumento de la temporalidad, la flexibilidad y un aumento considerable del desempleo, entre otros aspectos destacables (Miret y Vidal, 2007). Esta situación genera en el individuo un alto grado de incertidumbre laboral. El CS puede jugar un papel clave en la generación de nuevas oportunidades de empleo o en el acceso a determinada información (Granovetter, 1974; Burt, 2003).

Desde el estudio del CS a través de una perspectiva más próxima a la atributiva, autores como Toledo y Bastourre (2006) y Requena (1991, 1994) ponen de manifiesto la importancia de las relaciones para conseguir mejores oportunidades de acceso al empleo. Algunos estudios argumentan que los mecanismos 
tradicionales y formales de acceso al empleo, por méritos, por anuncios en el periódico, en internet, a través de bolsas de trabajo, etc. se ven fuertemente trastocados en épocas de fuerte crisis económica. Es en estos momentos de crisis cuando más se potencia el acceso al mercado de trabajo a través de mecanismos informales como son las relaciones sociales, contactos, información, recomendación, etc. (Ibáñez, 1999; Canteros y Espinoza, 2001).

En este contexto y para el estudio del CS en el mercado de trabajo catalán se presenta a continuación la definición del concepto de Trayectoria Laboral utilizada en este artículo. Uno de los aspectos fundamentales del concepto es que nos permite vincular las características individuales (micro) con los elementos estructurales que dibujan la coyuntura económica y la dinámica del mercado de trabajo (macro).

La perspectiva desde la cual se define el concepto de TL viene dada por un modelo temporal que se centra en la idea de proceso, en el cual, es imprescindible vincular la historia laboral del individuo con sus historias familiares y educativo-formativa (Godard, 1998; Muñiz, 2005). Además, cabe considerar otros factores que recogen la historia o recorrido relacional (antiguos conocidos, nuevos contactos, etc.) del individuo y que adquieren importancia respecto al acceso al empleo principalmente para colectivos como los jóvenes (Merino y García, 2007).

Entendemos la TL como un proceso en el que se va dando una sucesión de acontecimientos que consisten en el cambio de las posiciones que se ocupan en el mercado de trabajo. Es decir, este proceso se entiende como la vida o historia laboral del individuo. Se trata de secuencias de cambio que dibujan la dinámica de la TL en el tiempo y en el espacio. Estos cambios van asociados a una estructura de oportunidades en la que el individuo se encuentra inmerso (Figura 2).

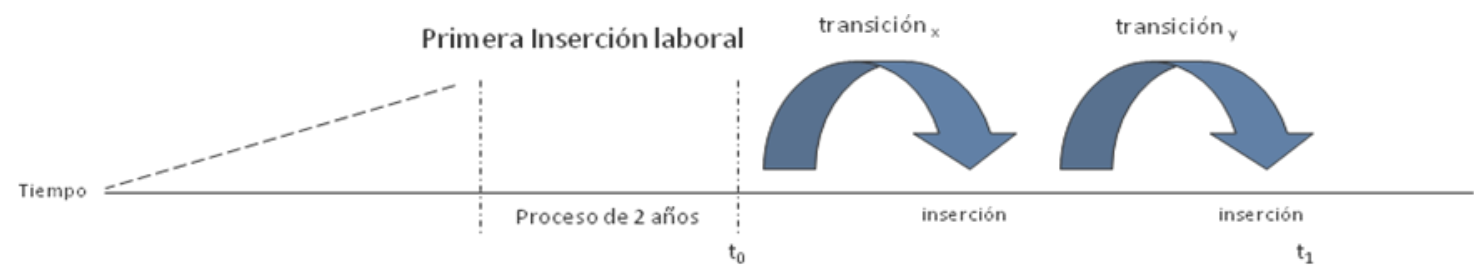

Historia residencial-familiar

Historia educativo-formativa

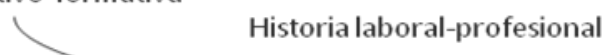

Figura 2. Representación de la definición del concepto de TL. 
En este contexto de la $\mathrm{TL}$, la importancia del CS radica en hacer posible el cambio de la situación laboral de los individuos, en forma de nuevas oportunidades de empleo. Así, la TL puede entenderse además como un proceso en el cual se van acumulando diversos recursos que pueden ser causa o pueden explicar los cambios (ascendentes o descendentes) en las posiciones que el individuo ocupa en el mercado de trabajo.

\section{La articulación de las perspectivas atributiva y reticular a través de los datos empíricos}

Para dar cuenta de nuestro modelo de análisis sobre trayectorias laborales y capital social desarrollamos un diseño articulado donde se combinan datos atributivos y datos reticulares. La información atributiva proviene de la Muestra Continua de Vida Laborales (MCVL) de la Seguridad Social, para el año 2007, y se analiza en un primer momento con el objetivo de obtener una tipología de posiciones y trayectorias en el mercado de trabajo con la que tipificar y validar los perfiles de los colectivos de estudio. Estos perfiles son definidos a priori en el modelo de análisis y son tratados específicamente en el análisis reticular. La información reticular se genera a partir de la Encuesta de Redes Personales (ERP) elaborada ad hoc para el proyecto de investigación mencionado, en el año 2010, y se analiza sobre la base de los tipos de trayectorias laborales de la MCVL con el objetivo de analizar los mecanismos relacionales de Cohesión Social e Integración Social.

\subsection{Definición de los colectivos de estudio}

Hemos definido y estudiado cinco colectivos de interés:
a) las personas nacidas en Cataluña de 55 a 64 años (C1: NC 55-64),
b) las personas nacidas en Cataluña de 25 a 54 años (C2: NC 25-54),
c) las personas nacidas en el resto de España de 55 a 64 años (C3: RE 55-64),
d) Ias personas nacidas en Ecuador de 25 a 64 años (C4: Ec 25-64), y
e) las personas nacidas en Marruecos de 25 a 64 años (C5: Ma 25-64). 
La definición de estos colectivos viene determinada principalmente por dos variables atributivas que ayudan a entender la posición del individuo en la estructura social ${ }^{3}$. La primera de ellas es el origen o lugar de procedencia de los individuos. Esta variable se considera pertinente ya que, en el contexto de la población que reside en Cataluña, podemos encontrar claras diferencias entre el colectivo de autóctonos e inmigrantes respecto a su situación en el mercado de trabajo, sus condiciones de vida, derechos, etc. En esta línea, y para contemplar la heterogeneidad que puede presentar el colectivo de población autóctona, se ha distinguido entre personas que han nacido en Cataluña y personas que han nacido en el resto de España (principalmente la inmigración de los años 50 y 60 procedente del resto del territorio español). En lo que se refiere al colectivo de inmigrantes procedentes de otros países, se ha considerado pertinente estudiar el colectivo de ecuatorianos y el de marroquíes ya que constituyen dos de los colectivos más representados en la composición de la población catalana. Además resultan interesantes por las propias diferencias existentes entre ellos y las diferencias en relación a los autóctonos, en términos culturales, de hábitos de vida, etc.

La segunda variable atributiva que define los colectivos de estudio es la edad. A través de ella se intenta diferenciar el periodo de plenitud en relación al mercado de trabajo y captar las diferencias generacionales en el acceso al empleo y los niveles educativos, además de tener en cuenta el aumento de la edad en el acceso al primer empleo (Casal, 2003).

La Encuesta de Redes Personales ha sido diseñada específicamente teniendo en cuenta los cinco colectivos. Posteriormente, estos se han identificado en la Muestra Continua de Vidas Laborales del 2007 para el estudio de las TL y para hacer posible la comparación y la validación entre los dos tipos de información de diferente naturaleza, la atributiva (MCVL) y la de carácter relacional (ERP) ${ }^{4}$.

\footnotetext{
${ }^{3}$ Los criterios para la construcción de los colectivos vienen del análisis previo de la muestra para Cataluña en la Encuesta de Condiciones de Vida y Hábitos de la Población de Cataluña 2006 (LópezRoldán y Lozares, 2008a; López-Roldán y Lozares 2008b).

4 Originariamente se trabaja con seis colectivos en el diseño de la Encuesta de Redes Personales 2010 ya que, además de la edad y el origen, también se tiene en cuenta el origen de los padres (si han nacido en Cataluña o en el resto de España). Consideramos tan sólo a cinco debido a la imposibilidad de identificar el origen de los padres en la base de datos atributiva de la Muestra Continua de Vidas Laborales de 2007.
} 
Además de la construcción y el manejo de estos colectivos en términos de origen y de sus edades correspondientes, se han añadido otros criterios para este análisis que están directamente vinculados a la temática de mercado de trabajo. Estos criterios son: (a) se trata única y exclusivamente de población en edad activa, (b) mantiene una relación de tipo laboral con la Seguridad Social durante el año 2007, y (c) se trata de población en situación asalariada.

\subsection{Presentación de los datos para el análisis}

El estudio de las TL se realiza a partir de la MCVL. Esta base de datos proporciona información de carácter puramente atributivo sobre las condiciones y la situación en la que se encuentran los individuos en relación al mercado de trabajo (García y Durán, 2008; Durán, 2007; Toharía y Cebrian, 2007; López-Roldán, 2011).

El análisis de los mecanismos relacionales de CoS e InS se realiza a partir de la ERP. Se trata de una base de datos de carácter reticular en la que se incluyen variables atributivas relativas a diversos ámbitos de la vida laboral, asociativa, familiar, etc. de los individuos encuestados (Ego) y de las personas nombradas por ellos (Alteri).

La MCVL recoge una muestra representativa de la población española, aunque nuestro trabajo se centra en la muestra para Cataluña, también representativa (un total de 68.615 casos). Esta base de datos proporciona registros administrativos con información sobre todas las relaciones laborales y no laborales de las personas que a 1 de abril de 2008 (fecha de extracción de la muestra) tenían algún tipo de relación con la Seguridad Social. Además de esta información, contiene todos los registros históricos anteriores a la fecha de extracción. Este aspecto será de suma importancia para contemplar la dimensión que recoge el carácter dinámico de la TL.

A partir de la MCVL se ha construido el proceso de dimensionalización del concepto de TL y se han realizado los análisis pertinentes para construir una Tipología de Trayectorias Laborales (López-Roldán, 1996a) para cada uno de los colectivos estudiados. El procedimiento se ha llevado a cabo a partir de la aplicación de diferentes técnicas multivariadas (Domínguez y López-Roldán, 1996) 5 .

En primer lugar se ha realizado un Análisis Factorial de Correspondencias Múltiples (ACM) que ha permitido: (a) contemplar los efectos de múltiples variables cualitativas de manera simultánea, (b) reducir la información disponible de las

5 El procesamiento de los datos de las dos matrices y la aplicación de las técnicas de análisis correspondientes se han llevado a cabo a través de los programas informáticos SPSS y SPAD respectivamente. 
variables originales en forma de factores de diferenciación que combinan la información más relevante para el estudio del colectivo en cuestión. En segundo lugar se ha realizado un Análisis de Clasificación Automática o de Conglomerados $(A C L)$ a partir de las variables factoriales para obtener una tipología. De esta forma se han identificado los perfiles de TL más representativos de cada uno de los cinco colectivos en el contexto del mercado de trabajo catalán.

La ERP recoge las características tanto atributivas como reticulares de los Egos y de sus Alteri, además de características estructurales de las redes personales (indicadores de Degree, Closenness, Betweenness, Cliques, etc.). Esta base de datos es resultado de la aplicación de 450 encuestas Ego-centradas (se trata de una muestra por cuotas, no estrictamente aleatoria y proporcional en términos poblacionales) en las que cada Ego tenía que nombrar a 30 Alteri para configurar su entorno relacional, además de establecer si los Alteri mencionados tenían o no relación (se conocían) entre ellos. Esta base de datos no permite construir el concepto de TL tal y como aquí se define, puesto que recoge información de carácter sincrónico. Sin embargo su contenido resulta fundamental para obtener información sobre CS en general y sobre sus tipos (Bonding Social Capital y Bridging Social Capital) y mecanismos relacionales (CoS e InS) en particular.

Las técnicas utilizadas para el análisis de los tipos de CS y mecanismos relacionales vienen de la construcción de indicadores estadísticos para medir la CoS y la InS y del Análisis de Tablas de Contingencia que permite el cruce entre las características de los Egos y de los Alteri.

\subsection{La articulación de las dos fuentes de datos. En busca del equilibrio en los perfiles de la población}

Para poder llevar a cabo el procedimiento de articulación entre las bases de datos y establecer paralelismos entre las dos poblaciones se ha realizado un ejercicio validativo con el objetivo de constatar si los perfiles que se han obtenido a partir del análisis de TL en la MCVL se dan de manera semejante en la muestra que se recoge en la ERP.

El procedimiento de contraste se ha llevado a cabo en dos fases. La primera de ellas ha consistido en cruzar mediante Tablas de Contingencia, la categoría profesional de los Egos con el origen de los mismos (cinco colectivos de estudio). La tendencia observada en cuanto a los resultados de las TL (elaborados a partir de la MCVL) y los resultados del cruce entre categoría profesional de los Egos y sus orígenes (elaborados a partir de la ERP) muestran la misma tendencia en cuanto a las características laborales de los individuos según sus colectivos de origen. 
En la Tabla 1 se presentan los resultados del ejercicio exploratorio realizado a partir de los datos de la ERP. Comparando estos resultados con los obtenidos en el análisis de TL con los datos de la MCVL (se presentan en los siguientes apartados) podemos observar que en ambas muestras las situaciones laborales de los individuos según sus colectivos de origen expresan los mismos perfiles.

\begin{tabular}{|c|c|c|c|c|c|c|c|}
\hline & & C1: NC 55-64 & C2: NC 25-54 & C3: RE 55-64 & C4: Ec 25-64 & C5: Ma 25-64 & Total \\
\hline \multirow{2}{*}{ CP Alta } & $\mathrm{n}$ & 120 & 150 & 30 & 60 & 60 & 420 \\
\hline & $\%$ & 14,8 & 3,9 & 4,5 & 3,8 & 3,6 & 4,9 \\
\hline \multirow{2}{*}{ CP Media } & $\mathrm{n}$ & 300 & 2220 & 210 & 510 & 270 & 3510 \\
\hline & $\%$ & 37,0 & 58,3 & 31,8 & 32,7 & 16,4 & 41,3 \\
\hline \multirow{2}{*}{ CP Baja } & $\mathrm{n}$ & 390 & 1380 & 420 & 960 & 1290 & 4440 \\
\hline & $\%$ & 48,1 & 36,2 & 63,6 & 61,5 & 78,2 & 52,3 \\
\hline \multirow{2}{*}{$\begin{array}{c}\text { No } \\
\text { clasificable }\end{array}$} & $\mathrm{n}$ & 0 & 60 & 0 & 30 & 30 & 120 \\
\hline & $\%$ & 0,0 & 1,6 & 0,0 & 1,9 & 1,8 & 1,4 \\
\hline \multirow{2}{*}{ Total } & $\mathrm{N}$ & 810 & 3810 & 660 & 1560 & 1650 & 8490 \\
\hline & $\%$ & 100,0 & 100,0 & 100,0 & 100,0 & 100,0 & 100,0 \\
\hline
\end{tabular}

Fuente: Elaboración propia a partir de la ERP

Nota: En negrita se señalan aquellos porcentajes más elevados que caracterizan a los Egos de un origen y una categoría profesional determinada.

Tabla 1. Categoría profesional (CP) de Ego según su origen/colectivo.

La segunda fase de este ejercicio ha consistido en incorporar la variable del sexo al cruce entre la categoría profesional de los Egos y sus orígenes (colectivos). Se ha visto que esta variable juega un papel fundamental en los perfiles identificados en la tipología de TL (MCVL). En ambas matrices, la tendencia observada en cuanto a la situación laboral se da de la misma manera tanto en el caso de la población autóctona como en el caso de la población inmigrante.

\section{El tratamiento de los conceptos desde las bases de datos}

\subsection{El concepto de Trayectoria Laboral en la Muestra Continua de Vidas Laborales}

Como en este artículo, la gran mayoría de estudios que elaboran una Tipología de Trayectorias Laborales lo hacen articulando los resultados empíricos con construcciones teóricas previas más o menos elaboradas (Canteros y Espinoza 2001; Eckert y Mora, 2007; Zuribi, 2006; Toharia y Cebrián, 2007). Las clasificaciones derivadas de trabajos como los de Spilerman (en García y Gutiérrez, 1996) han sido de gran ayuda para identificar los tipos de trayectorias obtenidos en este análisis. 
A partir de las variables que contiene la MCVL de 2007 se han contemplado tres dimensiones del concepto de TL (Tabla 2). La primera está compuesta por cuatro variables sociodemográficas. Esta primera dimensión recoge las características relativas a la oferta de la mano de obra ${ }^{6}$. Las variables de edad y lugar de nacimiento han servido para identificar los cinco grupos a los que se les aplicó el cuestionario de redes personales en la MCVL. La segunda dimensión, compuesta por siete variables, enfatiza la perspectiva estática y recoge las características de la situación laboral del momento (2007). Por último, la tercera dimensión, con cuatro variables, parte de la perspectiva dinámica y de proceso. Esta recoge indicadores de cambio en la trayectoria que permiten observar aspectos como la movilidad, antigüedad, edad de inicio y estabilidad contractual en situación de contrato indefinido.

\begin{tabular}{|c|c|}
\hline Dimensión & Indicador \\
\hline \multirow{4}{*}{$\begin{array}{l}\text { Socio- } \\
\text { demográfica } \\
\text { (Variables } \\
\text { Ilustrativas) }\end{array}$} & Sexo: Varón/Mujer \\
\hline & Edad: $25-34 / 35-44 / 45-54 / 55-64$ \\
\hline & Lugar de nacimiento: Cataluña/ Resto España/ Ecuador/ Marruecos \\
\hline & Nivel educativo: Primaria/ Secundaria/ Batchillerato-FP superior/ Universitarios \\
\hline \multirow{7}{*}{$\begin{array}{l}\text { Estática (2007) } \\
\text { (Variables } \\
\text { Activas) }\end{array}$} & Tipo de contrato: Indefinido/ Temporal \\
\hline & Jornada laboral: Completa/ Parcial \\
\hline & $\begin{array}{l}\text { Sector de actividad: Agricultura-ganadería/ Industria/ Construcción/ Comercio/ } \\
\text { Hostelería/ Transporte/ Educación/ Banca y Seguros/ Administración Pública/ } \\
\text { Hogares y servicios personales/ Sanidad/ Otras actividades }\end{array}$ \\
\hline & $\begin{array}{l}\text { Tamaño de la empresa (no trabajadores): } 1-10 / 11-25 / 26-50 / 51-100 / 101- \\
250 / 251-500 / 501+\end{array}$ \\
\hline & Cotización anual: en quintiles \\
\hline & $\begin{array}{l}\text { Grupo de cotización: Ingenieros licenciados/ Ingenieros técnicos/ Jefes } \\
\text { administrativos/ Ayudantes no titulados/ Oficiales administrativos/ } \\
\text { Subalternos/ Auxiliares administrativos/ Oficiales de } 1^{a} \text { y } 2 \text { a/ Oficiales de } 3 a / \\
\text { Peones }\end{array}$ \\
\hline & Régimen de cotización: General/ Agrario/ Empleados del Hogar \\
\hline \multirow{4}{*}{$\begin{array}{l}\text { Dinámica } \\
\text { (Variables } \\
\text { Activas) }\end{array}$} & Años desde la 10 relación con la SS: Hasta $2 / 2-6 / 6-10 / 10-20 / 20+$ \\
\hline & $\begin{array}{l}\text { Seguridad (\% días de contrato indefinido del total de días contratado): Baja } \\
(<50 \%) / \text { Media }(50-80 \%) / \text { Alta }(>80 \%)\end{array}$ \\
\hline & $\begin{array}{l}\text { Cambio en el grupo de cotización (promoción): Descendiente/ No cambia/ } \\
\text { Promoción alta/ Promoción media/ Promoción baja }\end{array}$ \\
\hline & $\begin{array}{l}\text { Edad de la } 10 \text { relación laboral (acceso mercado de trabajo): 16-18/ 19-21/ 22- } \\
24 / 25-34 / 35+\end{array}$ \\
\hline
\end{tabular}

Fuente: Elaboración propia a partir de la MCVL.

${ }^{6}$ En el ACM realizado con SPAD estas variables sociodemográficas no forman parte del cálculo de los factores. Se trata de variables ilustrativas que muestran la posición de los individuos o colectivos en función de su perfil sociodemográfico. Las variables activas son las que generan directamente las dimensiones factoriales sintéticas obtenidas en el ACM. 
Tabla 2. Proceso de dimensionalización del concepto de TL en la MVCL 2007 y categorías de las variables utilizadas.

\subsection{El concepto de Capital Social en la Encuesta de Redes Personales}

El estudio del CS en la ERP se ha llevado a cabo a través de la identificación de los mecanismos de CoS e InS que presentan los Egos en relación a sus Alteri en función de la categoría profesional y para cada uno de los cinco colectivos. La identificación de estos mecanismos relacionales en el contexto de la situación laboral de los Egos nos permite observar cómo se distribuyen los tipos de Bonding Social Capital y Bridging Social Capital en el contexto de categorías profesionales diferenciadas (alta, media y baja).

De esta forma podemos conocer si los individuos pertenecientes a un colectivo determinado y a una categoría profesional determinada se encuentran en una situación de CoS o de InS. Esto implica que en sus redes tienden a relacionarse más con personas de su misma categoría profesional (contexto de CoS en el cual las relaciones se distribuyen siguiendo el modelo de tipo Bonding Social Capital y de carácter homofílico) o por el contrario, muestran una tendencia a la interacción con individuos que forman parte de una categoría profesional diferente (contexto de InS en el cual las relaciones se distribuyen siguiendo el modelo de tipo Bridging Social Capital y de carácter heterofílico).

Este último mecanismo relacional (InS) facilitaría a los individuos acceder a nueva información y por lo tanto no redundante en su entorno (Granovetter, 1973), con lo cual aumentan considerablemente las probabilidades de disponer de información sobre oportunidades de empleo que de otra manera les serían desconocidas.

La CoS se ha medido a partir del porcentaje de efectivos coincidentes en una casilla (en una Tabla de Contingencia) que cruza la misma categoría profesional para los Egos y los Alteri. Para medir la InS se ha tenido en cuenta el peso en la distribución de cada una de las diferentes categorías profesionales de los Alteri en una categoría profesional de Ego determinada ${ }^{7}$.

\section{Tipos de relaciones en los tipos de Trayectorias Laborales}

A continuación se presentan los resultados de la aplicación del modelo teóricometodológico expuesto en los apartados anteriores. En primer lugar se presenta la

\footnotetext{
${ }^{7}$ La construcción de los indicadores de CoS e InS puede consultarse con más detalle en Lozares, LópezRoldán, Verd, Martí, Bolíbar, Cruz y Molina "El análisis de la Cohesión, Vinculación e Integración sociales en las encuestas Ego-net", de este monográfico.
} 
Tipología de Trayectorias Laborales elaborada con la MCVL para cada uno de los cinco colectivos. En segundo lugar se presentan los resultados sobre mecanismos relacionales y tipos de CS en función de la situación laboral que presenta cada uno de los colectivos en la ERP y teniendo en cuenta las tendencias observadas en sus TL.

\subsection{Trayectorias Laborales en el mercado de trabajo catalán a partir de la Muestra Continua de Vidas Laborales}

El tamaño de la submuestra con la cual se ha realizado el análisis es de 68.615 individuos. En la Tabla 3 se presenta la distribución porcentual de cada uno de los cinco colectivos en la muestra para Cataluña de la MCVL.

\begin{tabular}{|c|c|}
\hline Colectivo & \% de casos \\
\hline C1: NC 55-64 & $7,1 \%$ \\
\hline C2: NC 25-54 & $79 \%$ \\
\hline C3: RE 55-64 & $7,7 \%$ \\
\hline C4: EC 25-64 & $2,6 \%$ \\
\hline C5: Ma 25-64 & $3,4 \%$ \\
\hline
\end{tabular}

Tabla 3. Distribución porcentual para los cinco colectivos de estudio en la muestra para Cataluña (MCVL).

De la realización de cinco ACM, uno para cada colectivo, se presentan en la Tabla 4 sus soluciones factoriales obtenidas.

\begin{tabular}{|l|l|}
\hline & \multicolumn{1}{|c|}{$\begin{array}{c}\text { Ejes factoriales en cada uno de los cinco Análisis Factoriales de } \\
\text { Correspondencias Múltiples }\end{array}$} \\
\hline C1: NC 55-64 & $\begin{array}{l}\text { F1 }(44,48 \%): \text { Antigüedad y estabilidad en el empleo (sexo y estudios) } \\
\text { F2 }(16,87 \%): \text { Movilidad y tamaño empresa (estudios) } \\
\text { F3 }(12,55 \%): \text { Situación económica y sector de actividad (sexo) }\end{array}$ \\
\hline & $\begin{array}{l}\text { F1 }(38,82 \%): \text { Estabilidad y condiciones salariales y de empresa (estudios) } \\
\text { F2 }(28 \%): \text { Antigüedad en el mercado de trabajo, jóvenes vs. mayores (sexo y } \\
\text { estudios) } \\
\text { F3 (10,37\%): Movilidad y posición en el mercado de trabajo en términos de } \\
\text { profesión/ocupación (sexo y estudios) }\end{array}$ \\
\hline C3: RE 55-64 25-54 & $\begin{array}{l}\text { F1 }(65,65 \%): \text { Antigüedad, movilidad y condiciones laborales } \\
\text { F2 }(16,81 \%): \text { Estabilidad, jornada y dedicación al trabajo }\end{array}$ \\
\hline C4: Ec 25-64 & $\begin{array}{l}\text { F1 }(77,51 \%): \text { Condiciones laborales y antigüedad (sexo y estudios) } \\
\text { F2 }(9,72 \%): \text { Jornada y categoría ocupacional, componente de años dedicados al } \\
\text { mercado de trabajo }\end{array}$ \\
\hline C5: Ma 25-64 & $\begin{array}{l}\text { F1 (57,61\%): Condiciones de empleo y movilidad } \\
\text { F2 (20,10\%): Antigüedad y estabilidad en el empleo }\end{array}$ \\
\hline
\end{tabular}

Tabla 4. Resultado del contenido de las dimensiones factoriales para cada uno de los grupos a partir de la MCVL.

La Tabla 4 nos muestra cómo en cada uno de los ACM las variables que hacen referencia a aspectos como la movilidad y la antigüedad resultan fundamentales a la hora de dar forma a las dimensiones factoriales. Esto es interesante y coherente con las características inherentes a los colectivos definidos. Además de contemplar las diferencias en términos de origen de las poblaciones estudiadas (autóctonos e inmigrantes), se contemplan aspectos como la edad, que resultan determinantes 
para configurar una situación laboral determinada en cuanto a la estabilidad y la antigüedad en el mercado de trabajo.

En el ACM realizado para el colectivo C1: NC 55-64, obtenemos tres factores principales que explican el $73,9 \%$ de la inercia total del modelo. Respecto a la primera dimensión ( $F 1$ ), se ubican en el extremo negativo todas aquellas posiciones que tienen que ver con situaciones de alta estabilidad laboral (alto porcentaje de seguridad contractual y situación como indefinido), categorías profesionales altas, promoción media-baja y niveles de estudios medios. En el extremo positivo de la dimensión encontramos poca estabilidad, situaciones de intermitencia laboral (temporalidad), categorías profesionales bajas, etc. Es importante señalar aquí cómo la tendencia hacia una situación de pertenencia al régimen de empleados del hogar y femenina adquiere fuerza en este extremo de la dimensión. En la segunda dimensión (F2) los estudios y la movilidad en el empleo adquieren gran relevancia. Se establece una asociación entre tener estudios superiores y promocionar de manera ascendente. La tercera dimensión (F3) supone un matiz interesante respecto al sexo y a las condiciones laborales en términos contractuales y salariales. La figura femenina aparece más asociada a una situación de temporalidad, mientras que la masculina aparece asociada a una situación de estabilidad.

Las tres dimensiones obtenidas en el ACM para el colectivo C2: NC 25-54 suman un total del $77,2 \%$ de la inercia. La composición de las dimensiones primera (F1) y tercera (F3) son muy semejantes a las del colectivo anterior, aunque aquí las categorías profesionales y los niveles de estudios son más elevados. Este aspecto puede estar directamente vinculado a que la población a la que se hace referencia es ligeramente más joven. En cuanto a la segunda dimensión (F2) nos muestra que en términos generacionales y de antigüedad se da una polarización entre dos posiciones: (a) aquellas que se caracterizan por el acceso al empleo en edades jóvenes y llevan más de 20 años en el mercado de trabajo y, (b) aquellas que se caracterizan por el acceso al empleo en edades más adultas y llevan menos años en el mercado de trabajo (en este perfil los niveles de estudios son ligeramente superiores). El sexo también guarda relación con estas posiciones ya que se observa un nivel de estudios superior en las mujeres más jóvenes. Este aspecto también queda reflejado en esta dimensión.

A través del ACM para el colectivo C3: RE 55-64 se obtienen dos dimensiones que representan el $82,5 \%$ de la inercia. Aquí la primera dimensión (F1) muestra la siguiente polarización: (a) las posiciones que tienen que ver con la condición femenina, estudios primarios y bajas categorías profesionales (principalmente 
relacionadas con el régimen de empleados del hogar) y salarios bajos (extremo negativo) y, (b) las posiciones vinculadas a la condición masculina, también con bajas categorías profesionales pero con estudios medios y algo más de promoción en el empleo (extremo positivo). La segunda dimensión (F2) recoge los matices en términos de temporalidad y parcialidad (más vinculada a las posiciones femeninas y con niveles de estudios bajos), y la estabilidad y jornadas completas (más vinculadas a las posiciones con niveles de estudios medios y trayectorias masculinas).

Para el estudio de la población inmigrante (ecuatoriana y marroquí) los resultados nos muestran diferencias importantes respecto a las posiciones de los ecuatorianos en relación a los marroquíes.

En el ACM realizado para el C4: Ec 25-64 se obtienen 2 dimensiones que recogen el $87,2 \%$ de la inercia. En el caso del ACM para el colectivo C5: Ma 25-54 las 2 dimensiones obtenidas representan el $77,7 \%$ de la inercia. El colectivo C4: Ec 2564 presenta unas posiciones muy semejantes al C3: RE 55-64, en cuanto a sus condiciones laborales. Con el matiz de que, al estar formado por población más joven, el C4: Ec 25-64 presenta diferencias en los niveles de estudios de hombres y mujeres (en algunos casos son ligeramente superiores a los del C3: RE 55-64) y en la antigüedad. En el colectivo C5: Ma 25-54, las diferencias respecto al anterior se acentúan. Las posiciones están muy polarizadas en función de los sectores de actividad de hogares y servicios personales, agricultura y construcción. Las categorías profesionales, así como las condiciones salariales se concentran generalmente en niveles medios y bajos. La situación laboral del colectivo C5: Ma 25-64 respeto al colectivo C4: Ec 25-64 es ligeramente más precaria y en términos generales está caracterizada por peores condiciones laborales.

Partiendo de los ejes factoriales obtenidos en esta primera fase del análisis se han realizado cinco Análisis de Clasificación Automática $(A C L)$ utilizando las soluciones factoriales descritas para cada colectivo. Con este último procedimiento se han obtenido los clusters o grupos que configuran la Tipología de Trayectorias Laborales para cada colectivo. En la Tabla 5 aparece para cada colectivo su tipología correspondiente.

\begin{tabular}{|l|l|}
\hline & \multicolumn{1}{c|}{ Tipología de Trayectorias Laborales } \\
\hline \multirow{2}{*}{ C1: NC 55-64 } & $\begin{array}{l}\text { T1 }(21,7 \%): \text { Trayectorias profesionales, estables y cualificadas. Tendencia masculina } \\
\text { T2 }(\mathbf{5 7 , 2} \%): \text { Trayectorias estables de más baja cualificación. Tendencia masculina } \\
\text { T3 }(1,9 \%): \text { Trayectorias estables de más baja cualificación. Tendencia femenina } \\
\text { (principalmente más adultas que llevan mucho tiempo en el mercado de trabajo) } \\
\text { T4 }(19,1 \%): \text { Trayectorias temporales de cualificación media. Tendencia femenina } \\
\text { (principalmente más jóvenes con menos antigüedad en el mercado de trabajo) }\end{array}$ \\
\hline \multirow{2}{*}{ C2: NC 25-54 } & $\begin{array}{l}\text { T1 }(19,3 \%): \text { Trayectorias profesionales de alta cualificación, promoción y seguridad } \\
\text { contractual. Tendencia masculina } \\
\text { T2 }(24 \%): \text { Trayectorias profesionales de alta cualificación con promoción media. }\end{array}$ \\
\hline
\end{tabular}




\begin{tabular}{|c|c|}
\hline & $\begin{array}{l}\text { Tendencia femenina } \\
\text { T3 }(\mathbf{2 8 , 4 \%}) \text { : Trayectorias de modelo antiguo, estables y seguras con baja promoción } \\
\text { y cualificación. Tendencia masculina } \\
\text { T4 }(\mathbf{2 8 , 3} \%) \text { : Trayectorias temporales de baja cualificación y sin promoción. } \\
\text { Tendencia femenina }\end{array}$ \\
\hline C3: RE 55-64 & $\begin{array}{l}\text { T1 (72,6\%): Trayectorias estables con cualificación y promoción media. Con } \\
\text { antigüedad. Tendencia masculina (llevan más de } 20 \text { años en el mercado de trabajo y } \\
\text { acceden desde muy jóvenes) } \\
\text { T2 }(22,1 \%) \text { : Trayectorias inestables con promoción y antigüedad. Tendencia } \\
\text { femenina } \\
\text { T3 }(5,3 \%): \text { Trayectorias seguras sin cualificación y del Régimen de Empleados del } \\
\text { Hogar }(99 \%) \text {. Tendencia femenina }\end{array}$ \\
\hline C4: Ec 25-64 & $\begin{array}{l}\text { T1 }(\mathbf{6 4 , 9 \% ) : ~ T r a y e c t o r i a s ~ c u a l i f i c a d a s , ~ t e m p o r a l e s ~ c o n ~ m o v i l i d a d . ~ T e n d e n c i a ~} \\
\text { femenina } \\
\text { T2 }(20,1 \%) \text { : Trayectorias de baja seguridad y baja promoción. Tendencia masculina } \\
\text { T3 }(14,9 \%) \text { : Trayectorias estables, de baja cualificación y del Régimen de Empleados } \\
\text { del Hogar. Tendencia femenina }\end{array}$ \\
\hline C5: Ma 25-64 & $\begin{array}{l}\text { T1 }(\mathbf{6 4 , 7 \% ) : ~ T r a y e c t o r i a s ~ d e ~ b a j a ~ c u a l i f i c a c i o ́ n ~} \sin \text { movilidad e inestables. De la } \\
\text { Construcción }(58 \%) \text {. Tendencia masculina } \\
\text { T2 }(12,3 \%) \text { : Trayectorias temporales y precarias. De la Agricultura (64\%). Tendencia } \\
\text { masculina } \\
\text { T3 }(18,3 \%): \text { Trayectorias profesionales con antigüedad } \\
\text { T4 }(4,7 \%): \text { Trayectorias de baja cualificación y precarias. Del Régimen de Empleados } \\
\text { del Hogar }(100 \%) \text {. Tendencia femenina }\end{array}$ \\
\hline
\end{tabular}

Tabla 5. Perfiles de las Trayectorias Laborales a partir de la MCVL.

El análisis ha puesto de manifiesto la importancia de dos variables en la construcción de los perfiles. Estas variables son el sexo y el sector de actividad. El impacto de la variable sexo para configurar los perfiles ha estado presente en todos los colectivos. En el caso de la variable sector de actividad, su impacto se ha detectado principalmente en los colectivos C4: Ec 25-64 y C5: Ma 25-64. Los colectivos formados por población inmigrante muchas veces, independientemente de su cualificación, se concentran en sectores de actividad como el de hogares y servicios personales, la construcción y la agricultura (esto es más notable en el colectivo C5: Ma 25-64). El criterio para especificar en las trayectorias una tendencia masculina o femenina ha consistido en que uno de los sexos superara el $50 \%$ de los casos en el grupo.

Para el colectivo C1: NC 55-64 obtenemos una Tipología de Trayectorias Laborales compuesta por cuatro perfiles (Gráfico 1 ). El perfil que representa un porcentaje mayor respecto a la población total del colectivo se corresponde con una trayectoria estable y de baja cualificación (T2, representa el 57,2\% de los casos). Este perfil esta compuesto por un $99 \%$ de individuos que pertenecen al régimen general y por un $65 \%$ de hombres. Predominan los contratos indefinidos y de jornada completa con un alto nivel de seguridad en términos contractuales. Se trata de individuos que acceden entre los 16 y los 21 años al mercado de trabajo y se caracterizan por tener estudios primarios $(37 \%)$ y secundarios (39\%). Presentan niveles de promoción baja $(37 \%)$ o descendente $(17 \%)$. Sus salarios son medio-bajos y se 
concentra en categorías profesionales medio-bajas, concretamente pertenecientes a los sectores de la industria (31\%) y de la construcción (10\%).

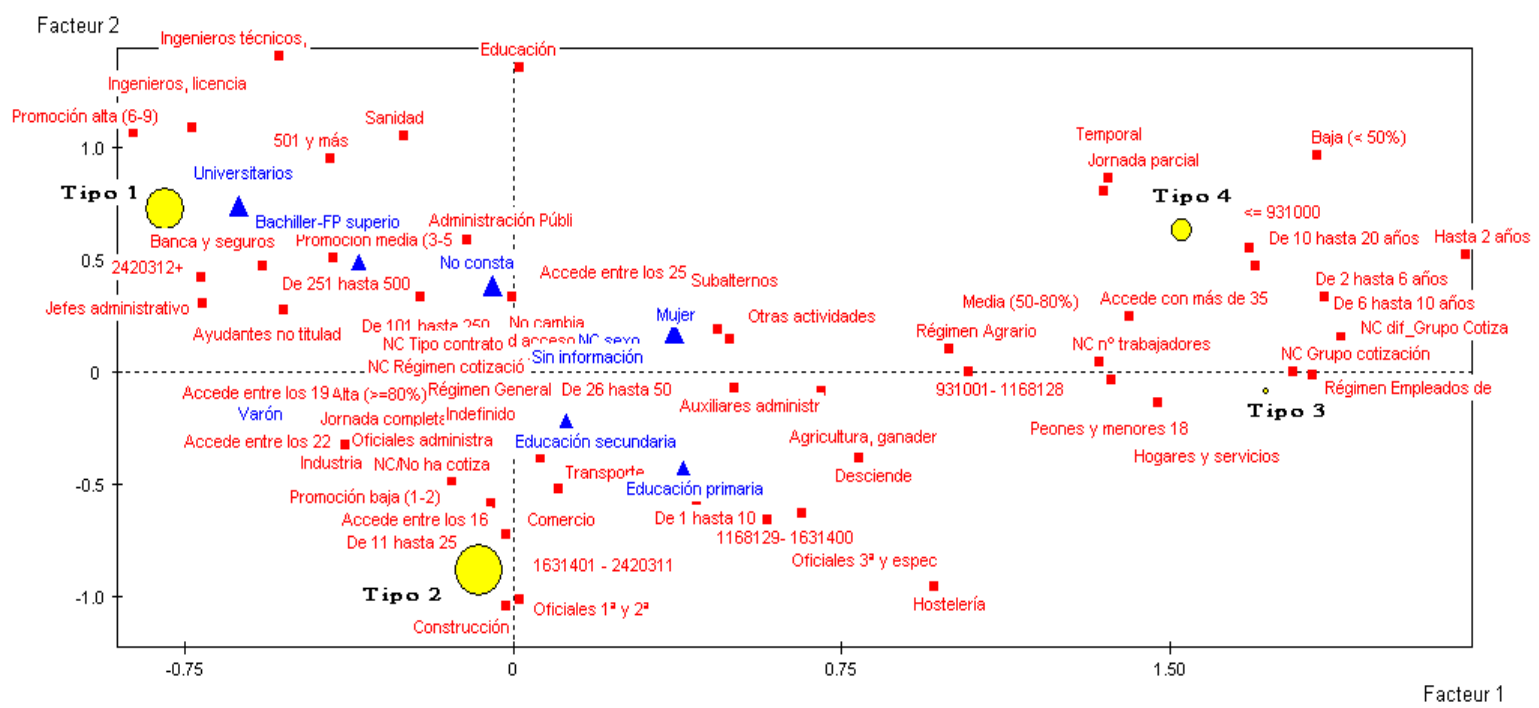

Gráfico 1. Gráfico factorial con las categorías de las variables ilustrativas (azul) y activas (rojo) y la Tipología de Trayectorias Laborales (clusters) para el colectivo C1: NC 55-64 a partir de la MCVL.

En el colectivo C2: NC 25-54 se han identificado cuatro perfiles (Gráfico 2). Los más característicos son dos: (a) la trayectoria de modelo antiguo y estable, segura con baja cualificación y promoción (T3, representa el 28,4\% de los casos) y (b) la trayectoria temporal de baja cualificación y sin promoción (T4, representa el 28,3\% de los casos). Mientras que la trayectoria de modelo antiguo guarda muchos paralelismos con el perfil característico del colectivo anterior (C1: NC 55-64, T2: fuertemente masculinizada, con contrato indefinido y jornada completa, etc.) la trayectoria temporal (T4) agrupa a los individuos con jornada parcial (43\%) y con contrato temporal (43\%). En la T4 la seguridad en términos contractuales es baja (39\%) y generalmente no se dan cambios en la promoción (40\%). El 68\% son mujeres con estudios primarios $(20 \%)$ y secundarios $(42 \%)$ y con salarios bajos (85\%). En este perfil encontramos categorías profesionales medio-bajas en sectores de actividad como el comercio (19\%), la sanidad (11\%) o la hostelería (7\%) y edades más jóvenes. Este efecto de la edad puede explicar la existencia de estas dos tendencias: (a) la trayectoria de modelo antiguo (T3), más característica de personas en edades más adultas y (b) la trayectoria temporal (T4) más característica de las personas jóvenes que acceden de manera intermitente al mercado de trabajo. 


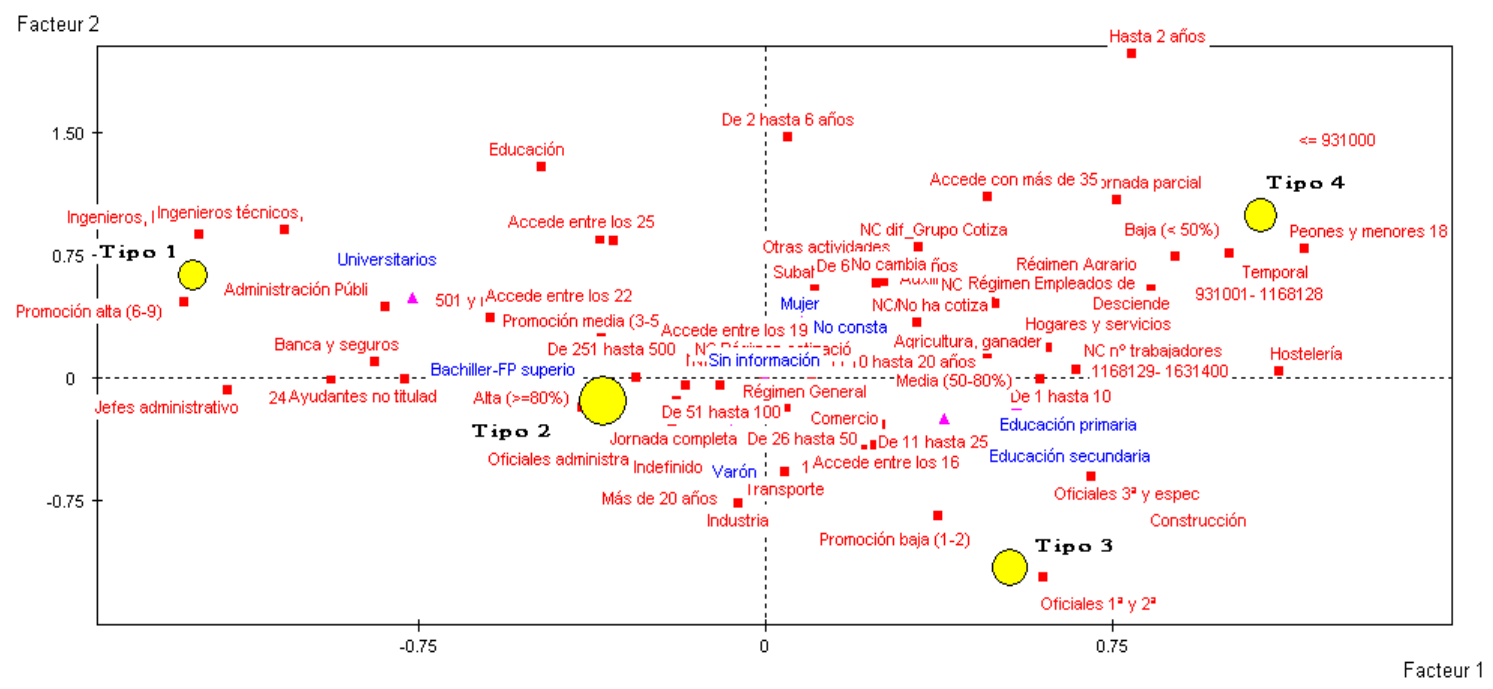

Gráfico 2. Gráfico factorial con las categorías de las variables ilustrativas (azul) y activas (rojo) y la Tipología de Trayectorias Laborales (clusters) para el colectivo C2: NC 25-54 a partir de la MCVL.

Para el colectivo C3: RE 55-64 se han obtenido tres tipos de TL (Gráfico 3). El perfil de trayectoria que se da en un porcentaje superior de individuos $(72,6 \%)$ es la estable, con cualificación y promoción media, antigüedad y de tendencia masculina (T1). Este perfil se caracteriza por el régimen general $(99,8 \%)$, la jornada completa $(98 \%)$ y el contrato indefinido (95\%). Presenta un alto nivel de seguridad contractual $(89 \%)$ y promoción baja $(35 \%)$ y media $(19 \%)$. El $76 \%$ de los casos son hombres y llevan más de 20 años (99\%) en el mercado de trabajo. Las categorías profesionales a las que pertenecen son medias y los principales sectores de actividad en los que se concentran son la industria (33\%), la construcción $(15 \%)$, el comercio (12\%) y el transporte (6\%). En este perfil se da una polarización entre aquellos individuos con estudios secundarios (30\%) que acceden muy jóvenes al mercado de trabajo (32\%), y aquellas personas con estudios de Bachillerato y FP superior que aceden a una edad adulta al mercado de trabajo $(40 \%)$. 


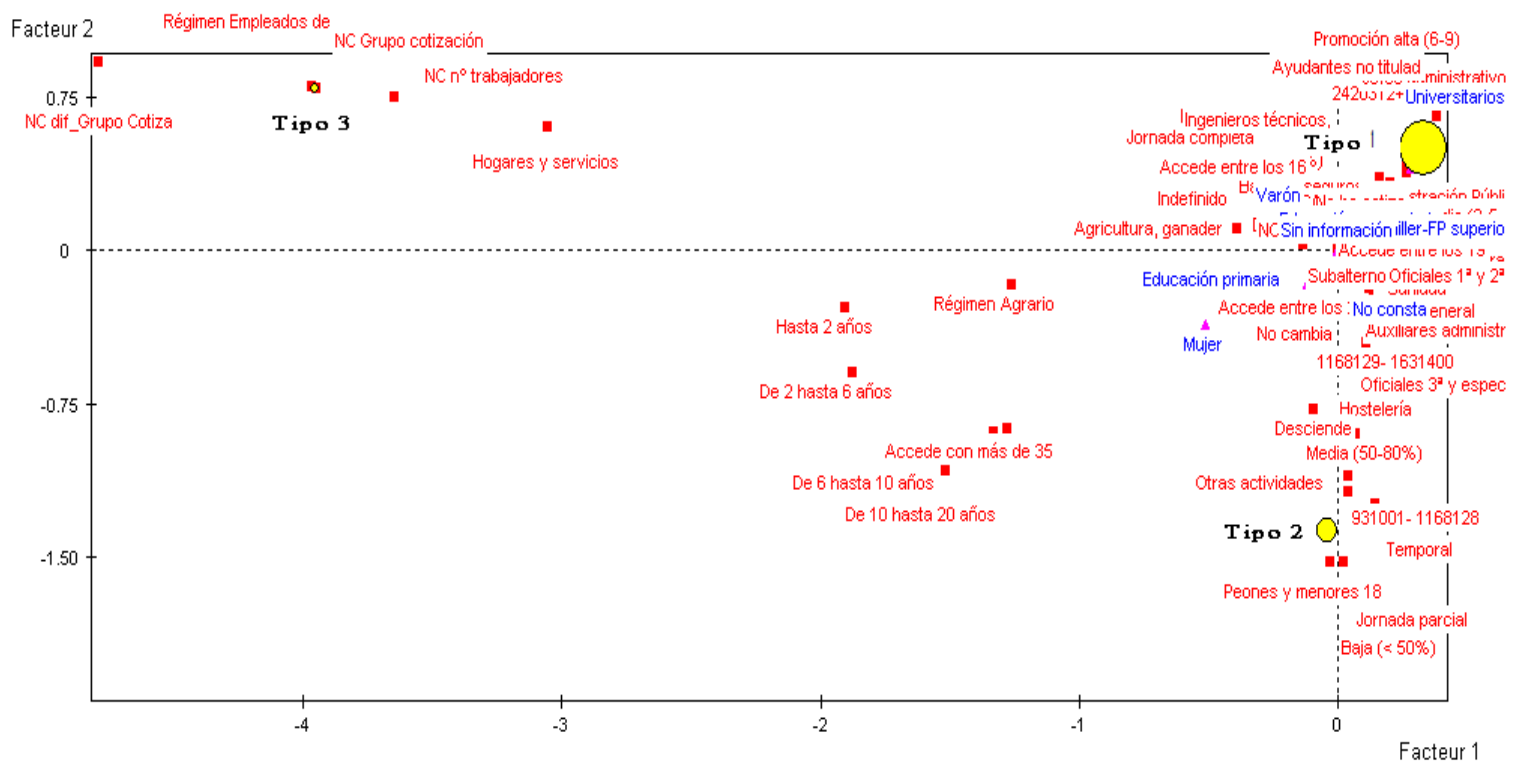

Gráfico 3. Gráfico factorial con las categorías de las variables ilustrativas (azul) y activas (rojo) y la Tipología de Trayectorias Laborales (clusters) para el colectivo C3: RE 55-64 a partir de la MCVL.

En el colectivo C4: Ec 25-64 se han identificado tres perfiles (Gráfico 4). El primer perfil (T1) es el más característico del colectivo $(64,9 \%)$ y muestra una tendencia femenina (67\% son mujeres). Agrupa a aquellos individuos en situación de temporalidad $(44 \%)$, con jornadas parciales (34\%) y baja seguridad contractual $(38 \%)$. Los salarios son bajos y los niveles de promoción son medios (13\%) o descendentes (15\%). En cuanto al nivel de estudios destaca un nivel de cualificación alto y la pertenencia a empresas con un gran número de trabajadores (22\%). Llevan entre 2 y 6 años en el mercado de trabajo (69\%) y se concentran en categorías bajas (51\%). El principal sector de actividad es el comercio (24\%). En términos generales, este colectivo muestra dos tendencias: (a) una hacia la inestabilidad y temporalidad, más característica de los grupos de riesgo, como las mujeres, los jóvenes y los inmigrantes y (b) otra tendencia más semejante al perfil más representativo del colectivo C3: RE 55-65 (T1: estable, con cualificación, etc.). 


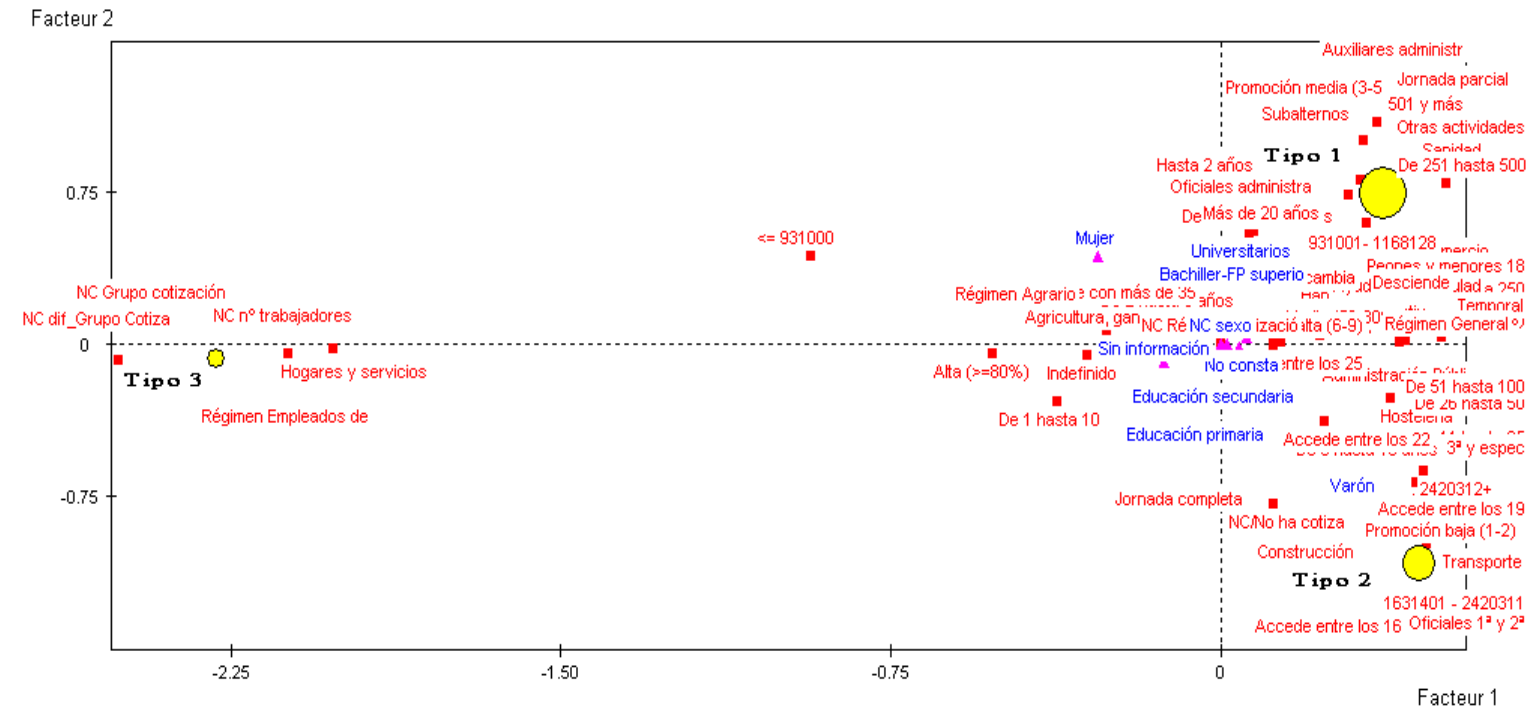

Gráfico 4. Gráfico factorial con las categorías de las variables ilustrativas (azul) y activas (rojo) y la Tipología de Trayectorias Laborales (clusters) para el colectivo C4: Ec 25-64 a partir de la MCVL.

Por último, para el colectivo C5: Ma 25-64 se han identificado cuatro perfiles (Gráfico 5). Este colectivo se encuentra en una situación laboral menos aventajada que el colectivo anterior en cuanto a su cualificación y temporalidad. Se concentra en determinados sectores de actividad o regímenes: construcción (58\%), agricultura (64\%) y régimen de empleados del hogar (100\%), de manera mucho más pronunciada que los colectivos anteriores. Desde la concepción de un mercado de trabajo segmentado vale decir que este colectivo se concentra en el fragmento o segmento secundario caracterizado por una situación laboral más precaria. De los cuatro perfiles identificados, aquel que agrupa un porcentaje superior de individuos es la trayectoria (T1) de baja cualificación sin movilidad e inestable $(64,7 \%)$. Este perfil va fuertemente vinculado al sector de actividad de la construcción (58\%) y su composición es principalmente masculina (85\%). El 75\% se encuentra en situación de temporalidad y se distribuyen de manera semejante entre jornada parcial y completa. No se dan cambios en cuanto a la promoción $(60 \%)$ y la seguridad contractual es baja (74\%). El 99\% pertenecen al régimen general y llevan entre 2 y 6 años en el mercado de trabajo (51\%) en categorías de peones (57\%). Los niveles educativos son más bajos (61\%) que los encontrados para el colectivo de ecuatorianos. 


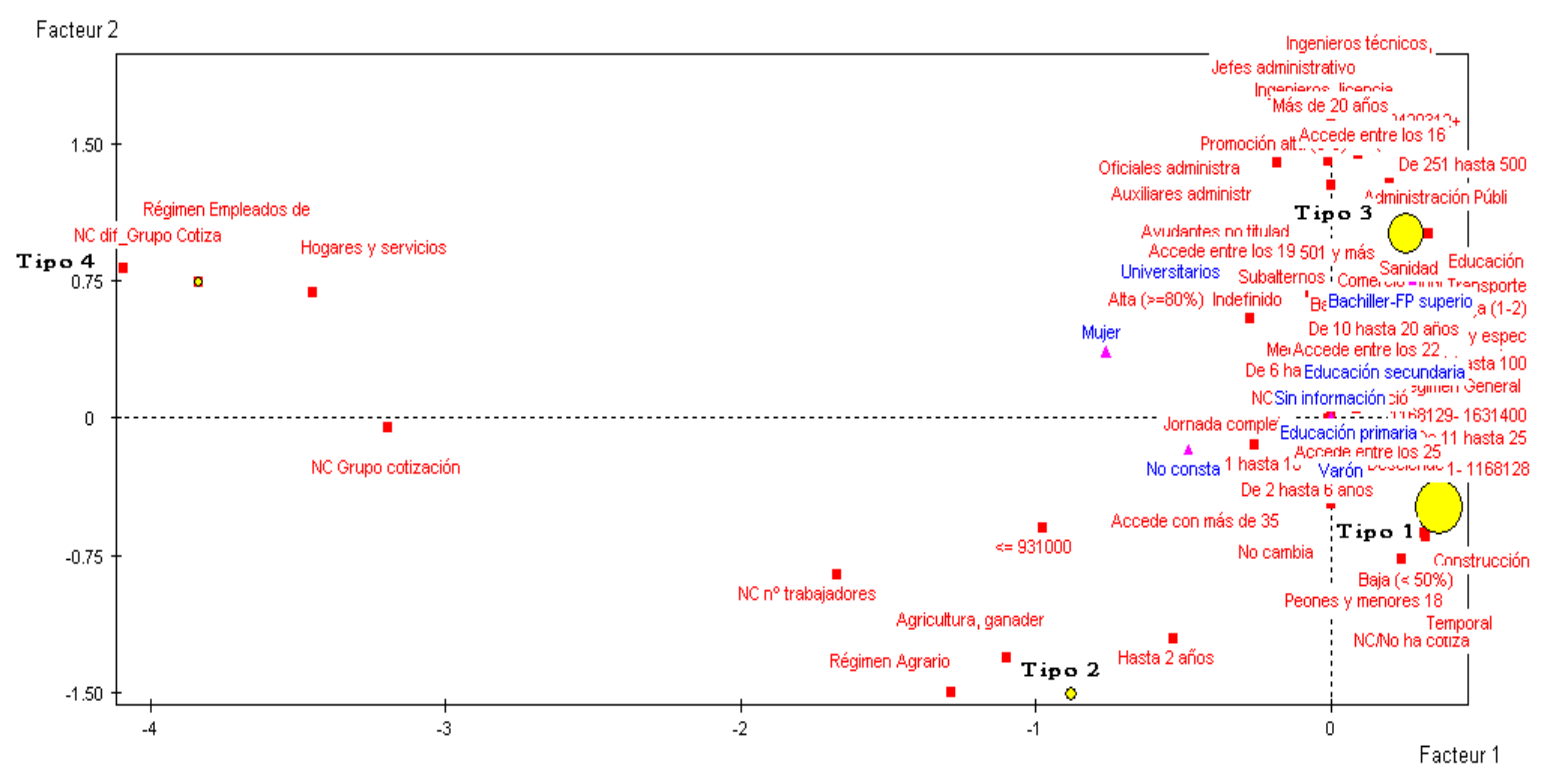

Gráfico 5. Gráfico factorial con las categorías de las variables ilustrativas (azul) y activas (rojo) y la Tipología de Trayectorias Laborales (clusters) para el colectivo C5: Ma 25-64 a partir de la MCVL.

\subsection{El apoyo deriva de la estructura. Los mecanismos de Cohesión e Integración a partir de la Encuesta de Redes Personales}

Teniendo en cuenta los perfiles de Trayectorias Laborales más representativos de cada colectivo construido a partir de la MCVL, ahora se pretende describir en la ERP qué tipos de relaciones se dan entre los Egos con esos perfiles (TL de la MCVL) y sus Alteri. Nos interesa identificar si los Egos se relacionan más con aquellos Alteri que pertenecen a su misma categoría profesional (situación de CoS en la que el tipo de CS más común es Bonding y la homofilia), o si por el contrario, se relacionan más con aquellos Alteri que pertenecen a categorías profesionales diferentes (situación InS en la que el tipo de CS más común es Bridging y la heterofilia). Este último tipo de CS puede facilitar a los Egos disponer de información que no se encuentra en su entorno laboral directo. Este aspecto puede aumentar las probabilidades de acceso a la información para generar nuevas oportunidades de empleo, y por tanto una mejor situación laboral (promoción).

El hecho de disponer de un perfil de TL general para cada colectivo (MCVL) nos permite ir a buscar en la ERP la misma tendencia observada respecto a la situación laboral de los Egos $^{8}$.

\footnotetext{
${ }^{8}$ Es importante recordar que el ejercicio para constatar si los perfiles coinciden en la MCVL y en la ERP se presenta en el segundo apartado de este artículo. Esto nos proporciona una mayor fiabilidad a la
} 
A continuación se presentan los resultados elaborados a partir de la ERP relativos a las formas de CS y a los mecanismos relacionales de CoS e InS.

Los indicadores que permiten extraer conclusiones sobre los niveles de CoS y de InS de un colectivo determinado se han elaborado a partir de una Tabla de Contingencia en la cual se cruza la categoría profesional de Ego (en columna) y categoría profesional los Alteri (en fila). El objetivo es ver, para cada categoría profesional de Ego, como se distribuyen las categorías profesionales de los Alteri. La Tabla 6 muestra el ejemplo para el colectivo C1: NC 55-64. En cuanto a la CoS este tipo de distribución nos permite ver si los Egos de una categoría profesional determinada se relacionan en mayor, o menor medida, con los Alteri de su misma categoría profesional (CoS) en la distribución de relaciones por columna. En cuanto a la InS la tabla nos permite calcular los valores medios o el peso que representan las categorías profesionales diferentes a las de Ego (sin tener en cuenta el porcentaje que recoge el nivel de CoS) ${ }^{9}$.

\begin{tabular}{|c|c|c|c|c|c|c|}
\hline \multirow{2}{*}{\multicolumn{3}{|c|}{ C1: NC 55-64 }} & \multicolumn{4}{|c|}{ CP EGO } \\
\hline & & & \multirow{2}{*}{$\begin{array}{c}\text { CP Alta } \\
39\end{array}$} & \multirow{2}{*}{$\frac{2 \text { CP Media }}{37}$} & \multirow{2}{*}{$\frac{3 \text { CP Baja }}{52}$} & \multirow{2}{*}{$\begin{array}{c}\text { Total } \\
128\end{array}$} \\
\hline \multirow{14}{*}{$\begin{array}{c}\text { CP } \\
\text { ALTER }\end{array}$} & \multirow{3}{*}{1 CP Alta } & $\mathrm{n}$ & & & & \\
\hline & & $\%$ & $32,50 \%$ & $12,30 \%$ & $13,30 \%$ & $15,80 \%$ \\
\hline & & Residuo corregido & 5,4 & $-2,10$ & $-1,90$ & \\
\hline & \multirow{3}{*}{2 CP Media } & $\mathrm{n}$ & 38 & 189 & 115 & 342 \\
\hline & & $\%$ & $31,70 \%$ & $63,00 \%$ & $29,50 \%$ & $42,20 \%$ \\
\hline & & Residuo corregido & $-2,5$ & 9,20 & $-7,10$ & \\
\hline & \multirow{3}{*}{3 CP Baja } & $\mathrm{n}$ & 28 & 56 & 179 & 263 \\
\hline & & $\%$ & $23,30 \%$ & $18,70 \%$ & $45,90 \%$ & $32,50 \%$ \\
\hline & & Residuo corregido & $-2,30$ & $-6,40$ & 7,90 & \\
\hline & \multirow{3}{*}{$\begin{array}{c}4 \text { No } \\
\text { clasificable }\end{array}$} & $\mathrm{n}$ & 15 & 18 & 44 & 77 \\
\hline & & $\%$ & $12,50 \%$ & $6,00 \%$ & $11,30 \%$ & $9,50 \%$ \\
\hline & & Residuo corregido & 1,20 & $-2,60$ & 1,70 & \\
\hline & \multirow{2}{*}{ Total } & $\mathrm{n}$ & 120 & 300 & 390 & 810 \\
\hline & & $\%$ & $100,00 \%$ & $100,00 \%$ & $100,00 \%$ & $100,00 \%$ \\
\hline
\end{tabular}

Fuente: Elaboración propia a partir de la ERP

Tabla 6. La CoS en la Tabla de Contingencia de C1: NC 55-64 según la Categoría Profesional (CP) de ego y la Categoría Profesional (CP) de los Alteri.

hora de trasladar las tendencias observadas respecto a un colectivo determinado y su situación en el mercado de trabajo desde la MCVL a la ERP.

9 Los indicadores se elaboraron en el contexto del proyecto citado con referencia CSO2008-01470. El indicador de InS se concreta en la siguiente fórmula: InS $(j): \sum_{i=2}^{\mathbb{P}}\left(X \mathrm{~b}_{\mathrm{j}}\right) / \mathrm{f} \mathrm{n}^{0}$ defilas , donde un valor del $100 \%$ indicaría la máxima InS. 
Para el colectivo $\mathrm{C} 1$ : NC 55-64 se da una tendencia a pertenecer principalmente a categorías profesionales medias y bajas (si observamos los totales para cada submuestra de la categoría profesional de $\mathrm{Ego}^{10}$ ), dándose de manera más intensa la pertenencia a esta última (tal y como se ha visto con la MCVL). Lo interesante de esta tabla es que nos permite ver que los Egos en categorías profesionales media y baja muestran unos niveles de CoS más elevados ( $63 \%$ y $45,9 \%$ respectivamente) que los Egos que pertenecen a categorías profesionales altas. Esto muestra que en la ERP, aquellas personas de categorías más bajas tienden a relacionarse más con personas que se encuentran en su misma categoría profesional. Por tanto esta tendencia implica relaciones homofílicas (entre individuos atributivamente semejantes) y de tipo Bonding Social Capital en un nivel de CoS más intenso para las categorías media y baja. En términos de oportunidades de acceder a información laboral que no viene de la misma categoría profesional, es decir, en términos de InS, los niveles más elevados para el colectivo C1: NC 55-64 los encontramos en la categoría profesional alta con un valor del 22,5\% (Tabla 8).

En las Tablas 7 y 8 se presenta de manera resumida los valores para la CoS y para la InS según las categorías profesionales de los Ego y los Alteri en cada colectivo. El procedimiento aplicado en los colectivos C2: NC 25-54, C3: RE 55-64, C4: Ec 25-64 y C5: Ma 25-64 es el mismo que para el C1: NC 55-54 (la Tabla de Contingencia).

\begin{tabular}{|c|l|c|c|c|c|c|}
\hline & $\begin{array}{c}\text { Origen } \\
\text { ego }\end{array}$ & C1: NC 55-64 & C2: NC 25-54 & C3: RE 55-64 & C4: Ec 25-64 & C5: Ma 25-64 \\
\hline \multirow{2}{*}{$\begin{array}{c}\text { CoS } \\
\text { según }\end{array}$} & Alta & $32,5 \%$ & $12 \%$ & $13,3 \%$ & $5 \%$ & $21 \%$ \\
\cline { 2 - 7 } & Media & $\mathbf{6 3} \%$ & $\mathbf{5 8 , 2} \%$ & $\mathbf{5 5 , 7} \%$ & $\mathbf{5 9 \%}$ & $38 \%$ \\
\cline { 2 - 7 } & Baja & $\mathbf{4 5 , 9 \%}$ & $\mathbf{5 2 , 5 \%}$ & $\mathbf{5 1 , 9} \%$ & $\mathbf{5 8 \%}$ & $\mathbf{5 6 \%}$ \\
\hline
\end{tabular}

Fuente: Elaboración propia a partir de la ERP

Tabla 7. Valor para el indicador del mecanismo de CoS según origen y categoría profesional (CP) de los Egos y los Alteri.

Para el C2: NC 25-54 vemos que las categorías profesionales en las que se agrupan con mayor intensidad los individuos son las categorías media y la baja (como en el grupo anterior). Uno de los elementos que hemos observado en el estudio de las TL para este colectivo es que el nivel de estudios y por tanto la posibilidad de pertenecer a categorías profesionales medias aumenta considerablemente respecto al colectivo anterior. Como vimos, este colectivo C2: NC 25-54 está formado por

\footnotetext{
10 La matriz de la ERP se estructura de la siguiente manera: en filas tenemos a los Egos y estos se repiten 30 veces (una por cada Alteri que ha nombrado el Ego). Esto requiere una lectura de los datos cautelosa, ya que el total de casos con los que trabajamos en la ERP no es de 810 (como muestra la Tabla 6), sino que es de 810/30. Esto nos da el resultado del no de Egos que configuran la muestra para este artículo (la población que actualmente está ocupada y asalariada).
} 
individuos más jóvenes y cualificados, esto puede ayudar a explicar una mejora en la categoría profesional de los Egos. Respecto a los niveles de CoS para este segundo grupo, se dan con una intensidad muy similar para las categorías media y baja ( $58,2 \%$ y $52,5 \%$ respectivamente).

Los niveles de CoS para el colectivo C3: RE 55-64 muestran una tendencia semejante, pero con porcentajes más bajos que el colectivo anterior. Los niveles de CoS para el tercer colectivo son de 55,7\% para la categoría profesional media, y de $51,9 \%$ para la baja. Los C4: Ec 25-64 y C5: Ma 25-64 constituyen el colectivo de origen inmigrante estudiado. Cabe señalar las diferencias que se dan entre ambos grupos según su origen. El colectivo C4: Ec 25-64, en términos de CoS, muestra una tendencia similar al de la población autóctona (este mismo fenómeno se ha observado también respecto a las TL), en cambio, el C4: Ma 25-64 presenta unos niveles de CoS muy elevados en la categoría profesional baja. Este último colectivo, en comparación con el resto, es el que se caracteriza más por relaciones fuertes (Bonding Social Capital) y orientadas hacia dentro de la comunidad.

\begin{tabular}{|c|c|c|c|c|c|c|}
\hline & $\begin{array}{c}\text { Origen } \\
\text { ego }\end{array}$ & C1: NC 55-64 & C2: NC 25-54 & C3: RE 55-64 & C4: Ec 25-64 & C5: Ma 25-64 \\
\hline \multirow{2}{*}{$\begin{array}{c}\text { InS } \\
\text { según }\end{array}$} & Alta & $\mathbf{2 2 , 5 \%}$ & $\mathbf{2 9 , 3} \%$ & $\mathbf{2 8 , 9} \%$ & $\mathbf{3 1 , 7} \%$ & $\mathbf{2 6 , 1 \%}$ \\
\cline { 2 - 7 } & Media & $12,3 \%$ & $13,9 \%$ & $14,8 \%$ & $13,7 \%$ & $\mathbf{2 0 , 6 \%}$ \\
\cline { 2 - 7 } ego & Baja & $18 \%$ & $15,8 \%$ & $16 \%$ & $13,9 \%$ & $14,7 \%$ \\
\hline
\end{tabular}

Fuente: Elaboración propia a partir de la ERP

Tabla 8. Valor del indicador del mecanismo de InS según origen y categoría profesional (CP) de los Egos y de los Alteri.

En lo que respecta al mecanismo de InS, en la Tabla 8 se presentan los valores para cada colectivo. Los resultados nos indican que, de la población autóctona, el colectivo mejor integrado en términos relacionales es C2: NC 25-54. De los Egos que forman este colectivo, aquellos con categorías profesionales altas son los que presentan un porcentaje superior de InS (29,3\%). Respecto a la población inmigrante, el colectivo mejor posicionado en términos de InS es el de los ecuatorianos con categorías profesionales altas $(31,7 \%)$. Teniendo en cuenta los perfiles laborales (Tipología de Trayectorias Laborales a partir de la MCVL) para los colectivos C2: NC 25-54 y C4: Ec 25-64, sus categorías profesionales y sus porcentajes de InS, podemos deducir que en términos de categoría profesional las relaciones de tipo Bridging Social Capital y de carácter heterofílico van asociadas a niveles de cualificación y categorías profesionales superiores. Esta situación de InS aumenta las oportunidades de disponer de información que no pertenece al entorno directo del individuo ya que circula por relaciones "que tienden puentes". 


\section{Conclusiones}

El análisis realizado a partir de un diseño de articulación de dos bases de datos, la Muestra Continua de Vidas Laborales de 2007, de carácter atributivo, y la Encuesta de Redes Personales de 2010, de carácter reticular, nos ha permitido extraer conclusiones respecto a los mecanismos relacionales de CoS e InS que presentan los colectivos estudiados según la posición que ocupan en el mercado de trabajo.

La observación de los perfiles laborales de la población autóctona e inmigrante que actualmente reside en Cataluña se ha concretado en el estudios de cinco colectivos. Estos han sido construidos a partir de sus edades y su lugar de nacimiento, y han sido objeto de interés ya que se esperaba encontrar diferencias relevantes respecto a sus Trayectorias Laborales.

Los cinco análisis tipológicos con la MCVL, uno para cada colectivo, han revelado diferencias en cuanto a los tipos de Trayectorias Laborales obtenidas. Estas diferencias no se han dado sólo entre la muestra de población autóctona y la muestra de población inmigrante, sino que además nos han permitido observar (a) la importancia de la edad en cuanto a las condiciones laborales y posición en el mercado de trabajo y en cuanto a los niveles de cualificación, (b) diferencias significativas en las Trayectorias Laborales que caracterizan los colectivos de autóctonos y de inmigrantes. Estas diferencias se centran, principalmente, en aquellos aspectos que tienen que ver con la promoción en el empleo y la movilidad, con la estabilidad y con la categoría profesional, en la que los grupos de autóctonos se encuentran mejor posicionados. Y (c) para la muestra de población inmigrante se ha observado que el colectivo de ecuatorianos se caracteriza por Trayectorias Laborales más similares a las de la población autóctona, mientras el colectivo de marroquíes se caracteriza por situaciones más precarias y fuertemente vinculadas a sectores de actividad determinados, como el sector de hogares y servicios personales, la agricultura y la construcción.

En lo que se refiere a los mecanismos relacionales y tipos de relaciones también se han encontrado diferencias significativas según el origen del colectivo y las principales categorías profesionales ocupadas por éstos. Aquellos perfiles (tanto de autóctonos como de inmigrantes) que se encuentran en categorías profesionales más bajas tienden a presentar unos niveles de CoS más elevados respecto a los perfiles que se concentran en categorías profesionales altas. Esta situación de CoS (caracterizada por relaciones homofílicas y Bonding Social Capital) a nuestro entender, puede estar contribuyendo a disminuir el espacio laboral de acción de estos individuos. Esto genera que las probabilidades de que estos individuos puedan 
acceder a nuevas oportunidades de empleo que mejoren su situación laboral disminuyen considerablemente en un contexto en el que el nivel de CoS es muy elevado. Esto queda reflejado al observar conjuntamente los resultados de los análisis de Trayectorias Laborales en la MCVL y los resultados de sobre mecanismos relacionales en la ERP. La baja promoción en el empleo tiende a estar más vinculada a categorías profesionales más bajas y a contextos con un nivel más alto de CoS.

De manera inversa, los perfiles que se caracterizan por pertenecer a categorías profesionales más altas muestran niveles más elevados de InS. Esta situación puede facilitar el acceso a información procedente de personas que pertenecen a categorías profesionales diferentes a las de Ego y que por tanto, disponen de nueva información laboral generada en otros entornos relacionales.

En líneas futuras de investigación y con el objetivo de complementar este análisis sería interesante identificar aquellos individuos (Ego) de la ERP que se ajustan mejor al perfil de Trayectoria Laboral más representativa en cada uno de los cinco colectivos de estudio. Este procedimiento permitiría el estudio detallado de la composición de sus redes personales y ayudaría a entender las dinámicas de interacción que se generan en la estructura relacional, así como las estrategias para la activación de los recursos disponibles en las redes que pueden ser útiles para mejorar situación en el mercado de trabajo.

\section{Bibliografía}

Alós-Moner, R. (2008). "Segmentación de los mercados de trabajo y relaciones laborales. El sindicalismo ante la acción colectiva". Cuadernos de Relaciones Laborales, vol. 26, no 1, pp 123-148.

Bourdieu, P. (1986). "The forms of capital", en Richardson, J. G. (ed.) Handbook of theory and research for the sociology of education. Nueva York: Greenwood, pp 241-258.

Burt, R. S. (1997). "The contingent value of social capital". Administrative Science Quarterly, n042 (2), pp 339-365.

Burt, R. S. (2003). "Structural holes and good ideas". American Journal of Sociology, 108, pp 1175-1210.

Canteros, E. y Espinoza, V. (2001). "Contactos sociales y carreras laborales en hogares chilenos de escasos recursos". Proposiciones n032, Santiago: Ediciones SUR, pp 170-189.

Casal, J. (2003). "De la escuela al trabajo". Cuadernos de Pedagogía no 326, pp 8186. 
Coleman, J. S. (1988). "Social capital in the creation of human capital", American Journal of Sociology, vol. 94, pp S95-S120.

Domínguez, M. y López-Roldán, P. (1996). "La construcció de tipologies: procés i tècniques d'anàlisi de dades". Papers n048, pp 31-39.

Durán, A. (2007). "La Muestra Continua de Vidas Laborales de la Seguridad Social", Revista del Ministerio de Trabajo y Asuntos Sociales, no 1, pp. 231-240.

Field, J. (2008). Social Capital. New York: Routledge.

Frane, A.; Roncevic, B. (2003). "Social capital: recent debates and research trends" Social Science Information, no42 (2), pp 155-183.

García, J.M. y Gutiérrez, R. (1996). "Inserción laboral y desigualdad en el mercado de trabajo: cuestiones teóricas." REIS, n 75/97, pp 269-293.

García, F. y A. Durán (2008). "Nuevos avances en la información laboral: la Muestra Continua de Vidas Laborales", Economistas, nº 116, pp 228-231.

Godard, F. (1998). "El debate y la práctica sobre el uso de las historias de vida en las ciencias sociales". Cuadernos del CIDS, Serie I Uso de las Historias de Vida en las Ciencias Sociales, Bogotá, Universidad Externado de Colombia.

Granovetter, M. S. (1973). "The strength of weak ties". American Journal of Sociology, vol. 78, n6, pp 1360-1380.

Granovetter, M. S. (1974). Getting a job. A study of contacts and careers. The University of Chicago Press, USA.

Hanifan, L. J. (1916). "The Rural School Community Center" Annals of the American Academy of Political and Social Science, n067, pp 130-138.

Herreros, F. (2002). "¿Son las relaciones sociales una fuente de recursos? Una definición del capital social", Papers, n67, pp 129-148.

Ibáñez, M. (1999). "El uso de las relaciones sociales en el acceso y mantenimiento del empleo" Revista Internacional de Sociología. Tercera Época, n²2, enero-abril, pp 129-152.

Lin, N. (2001). Social Capital: a theory of social structure and action. Cambridge: Cambridge University Press.

López-Roldán, P. (1996a). "La construcción de tipologías: metodología de análisis". Papers no 48, pp 9-29.

López-Roldán, P. (1996b). «La construcción de una tipología de segmentación del mercado de trabajo». Papers, no 48, pp 41-58.

López-Roldán, P. y Lozares, C. (2008a). "Implicaciones sociológicas en la construcción de una muestra estratificada", Empiria, n¹4, pp 87-108.

López-Roldán, P. y Lozares, C. (2008b). "La construcción de la muestra. El trabajo de campo de la Encuesta de condiciones de vida y hábitos de la población de Catalunya" Metodologies i Recerques. IERMB, Barcelona, nº 1, pp 17-39 
López-Roldán, P. (2011). "La Muestra Continua de Vidas Laborales: posibilidades y limitaciones. Aplicación al estudio de la ocupación de la población inmigrante". Metodología de Encuestas, n013, pp 7-32.

Lozares, C. (1996). "La teoría de redes sociales". Papers, n048, pp 103-126.

Lozares, C. (2003). "Valores, campos y capitales sociales". Revista hispana para el análisis de redes sociales, n04, \#2, Jun. http://revista-redes.rediris.es

Lozares, C., Verd, JM., López-Roldán, P., Martí, J., Molina, J.L. (2011). "Cohesión, Vinculación e Integración sociales en el marco del Capital social", REDES-Revista hispana para el análisis de redes sociales, vol. 20 \#1 (http://revistaredes.rediris.es).

Merino, R. y García, M. (2007). "Itineraris de formació i inserció laboral dels joves a Catalunya". Informes Breus, n04. Barcelona: Fundació Jaume Bofill.

Miret, P. y Vidal, E. (2007). "Temporalidad en los contratos de los jóvenes inmigrantes en España". Papers de Demografía, n³13, pp 1-13.

Molina, J. L. (2001). El análisis de redes sociales. Una introducción. Barcelona: Bellaterra.

Molina, J. L. (2004). "La ciencia de las redes". Apuntes de Ciencia y tecnología, no11, 36-42.

Muñiz, L. (2005). El aporte al concepto de trayectoria laboral para el estudio de las vivencias de los extrabajadores de YPF. Reflexiones a partir de la práctica. Buenos Aires: 70 Congreso Nacional de Estudios del Trabajo.

Portes, A. (1998). "Social capital: its origins and applications in modern sociology". Annual Reviews, no 24, pp 1-24.

Putnam, R. D. (1993). "The prosperous Community: Social capital and public life". The American Prospect no13, pp 35-42.

Putnam, R. D. (2003). El declive del capital social. Barcelona: Nueva Galaxia Gutenberg, Círculo de lectores.

Requena, F. (1991). Redes sociales y mercado de trabajo. Elementos para una teoría del capital relacional. Madrid: CIS/Siglo XXI.

Requena, F. (2004). "El capital social en la Encuesta de Calidad de Vida en el Trabajo", Papers no 73, pp 11-26.

Toharia, L. y Cebrián, I. (2007). Los efectos hipotéticos de la reforma laboral de 2006: Un análisis basado en la Muestra Continua de Vidas Laborales de la Seguridad Social. Jornadas de Usuarios de la MCVL (FFDEA).

Toledo, F. y Bastourre, D. (2006). "Capital social y recomposición laboral en Argentina. Un análisis para el período 1995-2000". Convergencia, enero-abril, vol. 13, no 040, pp 141-171.

Woolcock, M. y Narayan, D. (2000). "Social Ccapital: implications for development theory", Research and Policy, The World Bank Research Observer, n015, pp 225251. 
Zubiri, J. (2006). Trayectorias sociolaborales: introducción metodológica a las técnicas longitudinales en economía del trabajo. Bilbao: XI Jornadas de Economía Crítica. 